\title{
Diagnostic Value of High-Resolution Computed Tomography Scan in COVID-19: Do We Need to Think Outside the Box?
}

Muhammad Sheharyar Khan ${ }^{1}$, Muhammad Bilawal Abbas Janjua ${ }^{1}$, Ali Murad Jamal ${ }^{1}$, Shehrbano Qaiser ${ }^{2}$, Aamna Attiq ${ }^{3}$, Arsalan Raza ${ }^{4}$, Mustafa Tauseef Razzaq ${ }^{4}$, Assadullah A. Bhatti ${ }^{4}$, Nitasha Afzal ${ }^{5}$, Aiman Zahra ${ }^{6}$

1. Internal Medicine, Rawalpindi Medical University, Rawalpindi, PAK 2. Internal Medicine, Foundation University Medical College, Islamabad, PAK 3. Internal Medicine, Rahbar Medical and Dental College, Lahore, PAK 4. Internal Medicine, Rashid Latif Medical College, Lahore, PAK 5. Radiology, University College of Medicine and Dentistry, Lahore, PAK 6. Radiology, Sargoda Medical College, Sargoda, PAK

Corresponding author: Ali Murad Jamal, alimuradjamal@hotmail.com

\section{Abstract \\ Background and objective}

The ambiguous nature and high infectivity of the coronavirus disease 2019 (COVID-19) have caused soaring morbidity and mortality worldwide. Real-time polymerase chain reaction (RT-PCR) is preferred for detecting COVID-19. However, its poor sensitivity and the emerging use of high-resolution CT (HRCT) scan for disease severity make the use of RT-PCR quite obsolete. In light of this, our study aimed to explore the beneficial role of HRCT and compare the HRCT findings across various patient demographics and parameters.

\section{Methods}

This cross-sectional study included 100 patients with clinical suspicion of COVID-19. All patients underwent a chest HRCT scan preceded by RT-PCR testing. We used the CT severity score (CTSS) of the chest to calculate disease severity. Demographical data and results of radiological findings were tabulated and compared across RT-PCR positivity, age, and gender. Independent samples t-test and chi-square test were used to analyze the data.

\section{Results}

Glass ground opacity was the most prevalent finding in $99 \%$ of the patients, followed by lymph node involvement, consolidation, and crazy-paving pattern. Pleural effusion was observed in only $10 \%$ of the patients while pericardial effusion and hiatal hernia were present in 5\%. In RT-PCR-positive patients, the posterior basal segment of the lower lobe of the right and left lungs were found to be dominantly involved; however, the upper and middle lobes of the right lung were more commonly involved than the left lung. The mean CTSS was significantly higher in patients aged above 50 years $(\mathrm{p}<0.001)$. The mean CTSS of RT-PCRnegative patients was higher than that of RT-PCR-positive patients ( 15.18 vs. $14.31, \mathrm{p}=0.537$ ).

Review began 06/07/2021 Review ended 06/12/2021 Published 06/23/2021

\section{Copyright 2021}

Khan et al. This is an open access article distributed under the terms of the Creative Commons Attribution License CC-BY 4.0., which permits unrestricted use, distribution, and reproduction in any medium, provided the original author and source are credited.

\section{Conclusion}

RT-PCR has a limited role in the diagnosis of COVID-19. The HRCT scan can detect typical COVID-19 findings even in patients with negative RT-PCR results. Moreover, the use of HRCT scan in determining the disease severity and extent of lung damage can lead to a better assessment of critically ill patients.

Categories: Radiology, Infectious Disease

Keywords: coronavirus disease 2019 (covid-19), real-time polymerase chain reaction (rt-pcr), high-resolution ct scan, diagnostic value, computed tomography severity score (ctss) of chest

\section{Introduction}

The coronavirus disease 2019 (COVID-19) pandemic has severely paralyzed healthcare systems worldwide [1]. The highly transmissible virus belongs to a family of respiratory viruses and was named severe acute respiratory syndrome coronavirus 2 (SARS-CoV-2) [2]. COVID-19 can present as an asymptomatic disease, symptomatic illness (cough, altered taste, anosmia, and breathlessness), or manifest as various complications including acute lung injury, atypical pneumonia, cardiac injury, acute renal injury, and functional impairment [3]. All these complications ultimately translate into an increased risk of morbidity and mortality.

For diagnosing COVID-19 in suspected patients, a myriad of laboratory and radiological diagnostic modalities are available. These modalities include real-time polymerase chain reaction (RT-PCR), serological 
testing through enzyme link immunosorbent assay (ELISA), chest radiography, and high-resolution CT (HRCT) scan [4]. Currently, WHO has recommended the use of RT-PCR over serological testing for detecting SARS-CoV-2 [5]. However, this test has certain limitations that include cumbersome laboratory workup, time-consuming tests, high expenses, shortage of kits, and a low sensitivity ranging between $60-71 \%[5,6]$. Chest HRCT scan has been proven to have a central role in detecting COVID-19 in patients with negative RTPCR; it has also created a benchmark in detecting the disease severity and is significant for monitoring the treatment efficacy and disease prognosis. Chest HRCT scan can detect various features including consolidation, ground-glass opacities, crazy-paving patterns, lobar and segmental involvement of lungs, involvement of mediastinal lymph node, and extra-pulmonary manifestations such as pleural or pericardial effusions $[5,7]$.

The CT severity score (CTSS) of the chest is used for assessing the disease severity and the extent of lung damage [8]. It includes radiological observation of the 20 regions of both lungs and notices maximally involved segments. The chest CTSS is defined as the sum of scores of each lung segmental region, ranging from $0-40$ according to the severity $[5,8]$. Based on these HRCT scan findings and chest CTSS, the disease severity in suspected COVID-19 patients is graded from very low (mild disease) to very high (critical illness) $[8]$.

In this study, we aim to explore and investigate the role of HRCT scan in COVID-19 while examining the prevalence of various pulmonary and extrapulmonary findings. We further aim to compare these findings among patients with positive and negative RT-PCR results. Lastly, we aim to assess the differences in mean CTSS across ages, genders, and RT-PCR positivity. The findings of our study would lead to the implementation of measures to ensure favorable outcomes in COVID-19 patients in terms of disease identification, stratification, treatment, prognosis, and assessment for severity.

\section{Materials And Methods}

This was a retrospective cross-sectional study conducted on 100 patients with clinical suspicion of COVID19. The study was conducted at the Department of Radiology, Citi Laboratories in Rawalpindi, Pakistan from February to April 2021. The inclusion criteria were as follows: suspected COVID-19 patients with typical symptoms (cough, shortness of breath, documented fever, and body aches), oxygen saturation below 94\%, and COVID-19 suspicion based on initial chest X-ray findings (peripheral pulmonary infiltrates and consolidation). The exclusion criteria consisted of patients having non-viral pneumonia, tuberculosis, asthma, chronic obstructive pulmonary disease, and pulmonary edema. These patients were excluded based on systemic examination and patient history. Data was collected using a self-designed proforma that included demographic details, RT-PCR results, and chest HRCT scan findings. All the patients underwent HRCT scans according to the standard protocol, and chest CTSS was calculated from HRCT scan findings.

The HRCT scan was obtained from a multidetector Toshiba Aquilion CT scanner (Canon Medical Systems Corporation, Otawara, Japan) with a tube voltage of $120 \mathrm{kbp}$ and a current of $350 \mathrm{~mA}$. The supine position was used to obtain the chest HRCT scan. All lung fields were completely assessed from apices to bases and a consultant radiologist (with experience of 10 years in the field) reported all the findings. Thereafter, chest CTSS was calculated from the HRCT scan findings, and findings in each lung segment were reported. Categorical variables were expressed as frequency and percentages, while continuous variables were described using mean \pm standard deviations. Independent samples t-test and chi-square test were used to analyze the data. A p-value of below 0.05 was considered statistically significant. Data were entered and analyzed using SPSS Statistics version 23.0 (IBM, Armonk, NY).

\section{Results}

Our cohort had a total of 100 patients; the mean age of the patients was $54.0 \pm 14.0$ years (range: $24-83$ years). Of these 100 patients, 55 (55\%) patients were male and 45 (45\%) were females. In patients with proven RT-PCR positivity, there was a significant association with consolidation $(88.5 \%$ vs. $63.6 \%, \mathrm{p}=0.006)$ and lobe involvement $(\mathrm{p}=0.001)$. Cardiomegaly was more common with patients aged more than 50 years ( $51.6 \%$ vs. $21.1 \%, \mathrm{p}=0.002$ ). The comparison of HRCT findings across RT-PCR positivity is shown in Table 1 . 


\section{Cureus}

\begin{tabular}{|c|c|c|c|c|}
\hline \multicolumn{2}{|l|}{ Feature } & RT-PCR-positive patients ( $n=78), n(\%)$ & RT-PCR-negative patients (n=22), n (\%) & Total $(n=100), n(\%)$ \\
\hline \multirow{3}{*}{ Dominant lobe involvement } & Right & 47 (60.3\%) & $3(13.6 \%)$ & 50 (50.0\%) \\
\hline & Left & 14 (17.9\%) & $0(0.0 \%)$ & 14 (14.0\%) \\
\hline & Equal & $17(21.8 \%)$ & $19(86.4 \%)$ & $36(36.0 \%)$ \\
\hline \multirow{3}{*}{ Segment involvement } & Upper & $5(6.4 \%)$ & $3(13.6 \%)$ & $8(8.0 \%)$ \\
\hline & Middle & $0(0.0 \%)$ & $0(0.0 \%)$ & $0(0.0 \%)$ \\
\hline & Lower & $73(93.6 \%)$ & $19(86.4 \%)$ & 92 (92.0\%) \\
\hline \multirow{2}{*}{ Ground-glass appearance } & Yes & $77(98.7 \%)$ & $22(100 \%)$ & $99(99.0 \%)$ \\
\hline & No & $1(1.3 \%)$ & $0(0.0 \%)$ & $1(1.0 \%)$ \\
\hline \multirow{2}{*}{ Consolidation } & Yes & $69(88.5 \%)$ & $14(63.6 \%)$ & $83(83.0 \%)$ \\
\hline & No & $9(11.5 \%)$ & $8(36.4 \%)$ & 17 (17.0\%) \\
\hline \multirow{2}{*}{ Crazy-paving pattern } & Yes & 59 (75.6\%) & $15(68.2 \%)$ & 74 (74.0\%) \\
\hline & No & $19(24.4 \%)$ & 7 (31.8\%) & $26(26.0 \%)$ \\
\hline \multirow{2}{*}{ Involvement of lym } & Yes & $76(97.4 \%)$ & 21 (95.5\%) & $97(97.0 \%)$ \\
\hline & No & $1(2.6 \%)$ & $2(4.5 \%)$ & $3(3.0 \%)$ \\
\hline \multirow{2}{*}{ Pleural effusion } & Yes & $6(7.7 \%)$ & $4(18.2 \%)$ & $10(10.0 \%)$ \\
\hline & No & 74 (92.3\%) & $18(81.8 \%)$ & $90(90.0 \%)$ \\
\hline \multirow{2}{*}{ Cardiomegaly } & Yes & 32 (41.0\%) & $8(36.4 \%)$ & $40(40.0 \%)$ \\
\hline & No & $46(59.0 \%)$ & $14(63.6 \%)$ & 60 (60.0\%) \\
\hline \multirow{2}{*}{ Hiatal hernia } & Yes & $5(6.4 \%)$ & $0(0.0 \%)$ & $5(5.0 \%)$ \\
\hline & No & 73 (93.6\%) & 22 (100.0\%) & 95 (95.0\%) \\
\hline \multirow{2}{*}{ Pericardial effusion } & Yes & $3(3.8 \%)$ & $2(9.1 \%)$ & $5(5.0 \%)$ \\
\hline & No & 75 (96.2\%) & 20 (89.9\%) & $5(95.0 \%)$ \\
\hline
\end{tabular}

\section{TABLE 1: Comparison of HRCT scan findings between RT-PCR-positive and RT-PCR-negative}

patients

RT-PCR: real-time polymerase chain reaction; HRCT: high-resolution computed tomography

The mean chest CTSS among the patients was $14.5 \pm 5.83$. The score showed a difference when stratified according to age above 50 years, gender, and RT-PCR positivity, as shown in Table 2. 


\section{Cureus}

\begin{tabular}{|c|c|c|c|}
\hline \multicolumn{2}{|l|}{ Parameter } & Mean chest CTSS \pm SD & P-value* \\
\hline \multirow{2}{*}{ Gender } & Male & $14.11 \pm 5.5$ & \multirow{2}{*}{0.462} \\
\hline & Female & $14.98 \pm 6.1$ & \\
\hline \multirow{2}{*}{ RT-PCR positivity } & Yes & $14.31 \pm 5.8$ & \multirow{2}{*}{0.537} \\
\hline & No & $15.18 \pm 6.2$ & \\
\hline \multirow{2}{*}{ Age above 50 years } & Yes & $16.21 \pm 5.1$ & \multirow{2}{*}{$<0.001$} \\
\hline & No & $11.71 \pm 4.8$ & \\
\hline
\end{tabular}

\section{TABLE 2: Comparison of chest CTSS across patient parameters}

*Independent samples t-test

CTSS: computed tomography severity score; RT-PCR: real-time polymerase chain reaction; SD: standard deviation

The posterior basal segments of the lower lungs showed the maximum level of involvement in RT-PCRpositive patients $(\mathrm{n}=78)$. This is illustrated in Table 3 .

\begin{tabular}{|c|c|c|c|}
\hline Lung lobe & Segment & Right, n (\%) & Left, n (\%) \\
\hline \multirow{5}{*}{ Upper } & Apical & $53(67.9 \%)$ & 36 (46.2\%) \\
\hline & Anterior & 46 (59.7\%) & 38 (48.7\%) \\
\hline & Posterior & 67 (85.9\%) & $52(66.7 \%)$ \\
\hline & Lingula-superior & $0(0.0 \%)$ & $53(67.9 \%)$ \\
\hline & Lingula-inferior & $0(0.0 \%)$ & 65 (83.3\%) \\
\hline \multirow{2}{*}{ Middle } & Lateral & 57 (73.1\%) & $0(0.0 \%)$ \\
\hline & Medial & 54 (69.2\%) & $0(0.0 \%)$ \\
\hline \multirow{5}{*}{ Lower } & Superior & $51(65.4 \%)$ & 50 (64.1\%) \\
\hline & Medial basal & 57 (73.1\%) & $51(65.4 \%)$ \\
\hline & Anterior basal & 48 (61.5\%) & 50 (64.1\%) \\
\hline & Lateral basal & 57 (73.1\%) & 59 (75.6\%) \\
\hline & Posterior basal & 70 (89.7\%) & 70 (89.7\%) \\
\hline
\end{tabular}

TABLE 3: A breakdown of lung involvement among 78 RT-PCR positive patients

RT-PCR: real-time polymerase chain reaction

\section{Discussion}

Our study showed that the most common features detected even in RT-PCR-negative patients were groundglass appearance and consolidation. A study from Pakistan has reported similar findings, with ground-glass appearance and consolidation among $88.5 \%$ and $52.8 \%$ of the patients respectively [5]. Similarly, a study conducted in Italy showed ground-glass appearance with or without consolidation in nearly $97 \%$ of the patients [9]. In our study, the crazy-paving pattern on HRCT scan was observed in $74 \%$ of the patients; however, other studies have reported its prevalence to be between $12.5-36 \%$ [10,11]. In our study, pleural and pericardial effusion were observed in $10 \%$ and $5 \%$ of the patients respectively. Other studies in the literature have also reported a similar prevalence: between 4.8-8.4\% [11,12]. The literature substantiates the involvement of the right lower lobe more than the left lung [5,11]. Our study showed that the posterior basal segments of the lower lobes of both lungs were predominantly involved followed by posterior segments of the upper lobe of the right lungs. These findings are in line with another study that showed that lateral basal 
and medial basal segments of the right lung were predominantly involved in moderate-to-critical COVID-19 patients [13].

Our study has shown that the chest HRCT scan can detect typical findings of COVID-19 even in patients with negative RT-PCR results. Non-contrast HRCT scan is now a preferred diagnostic modality and plays a more crucial role in the diagnosis of clinically suspected COVID-19 patients [14]. Similarly, many studies have established that HRCT scan has a higher sensitivity of up to $85 \%$ and specificity of up to $88 \%$ as compared to RT-PCR for diagnosing COVID-19 [8,14,15]. In China, the National Health Commission authorities have also recommended the use of HRCT scan over RT-PCT for COVID-19 diagnosis due to its high accuracy [16]. Unfortunately, developing countries like Pakistan have not yet caught up and health professionals here mostly rely on RT-PCR positivity due to a lack of equipment and qualified radiologists. Raising awareness among healthcare professionals and encouraging the use of RT-PCR results along with chest HRCT scan results might offer a better solution to the problem.

Patients suffering from COVID-19 present with a vast array of symptoms ranging from asymptomatic disease to life-threatening cytokine storm [7]. The cytokine storm damages the pulmonary system, which can be life-threatening, especially in patients with multiple comorbidities and advanced age [17]. The results of our study are consistent with this finding as older patients had significantly higher chest CTSS than younger patients. In our study, it was observed that patients with positive RT-PCR had a lower mean chest CTSS as compared to patients with negative RT-PCR results. A study from Egypt has also shown similar results, by reporting a non-significant difference across positive and negative RT-PCR results [18]. Another study from Pakistan on chest HRCT scan findings in patients with RT-PCR-negative results has reported that $79.2 \%$ of patients had ground-glass opacities [19]. In comparison, our study reported ground-glass appearance in 98.7\% of RT-PCR-positive COVID-19 patients and in 100\% of RT-PCR-negative COVID-19 patients.

The major limitation of our study was its retrospective cross-sectional design based on a convenience sampling technique. We recommend that further cohort studies be conducted to delve deeper into the diagnostic role of chest HRCT in COVID-19 so that its superiority over RT-PCR can be conclusively established. There is an urgent need for the implementation of guidelines so that the diagnostic accuracy of COVID-19 can be established in a better way.

\section{Conclusions}

Patients with RT-PCR-negative results may present with typical COVID-19 features on HRCT scan evaluation. Chest HRCT scan can delineate several pulmonary and extrapulmonary findings that aid in determining the disease severity. Upon HRCT scan evaluation, posterior basal and lateral basal segments of the lower lobe of the right lung were found to be dominantly involved. The comparative findings on HRCT scan and CTSS between positive and negative RT-PCR patients demonstrated non-significant differences. Hence, the use of a chest HRCT scan along with clinical correlations could offer a solution for low RT-PCR sensitivity in the diagnosis of COVID-19 and its severity.

\section{Additional Information \\ Disclosures}

Human subjects: Consent was obtained or waived by all participants in this study. Citi Laboratories IRB, Rawalpindi issued approval IRB-2021-RADIOL-003. The study entitled "Diagnostic Value of High Resolution Computed Tomography Scan in Coronavirus Disease 2019: Do We Need to Think Outside the Box?" has satisfied the IRB board of Citi Laboratories and is approved for data collection. Animal subjects: All authors have confirmed that this study did not involve animal subjects or tissue. Conflicts of interest: In compliance with the ICMJE uniform disclosure form, all authors declare the following: Payment/services info: All authors have declared that no financial support was received from any organization for the submitted work. Financial relationships: All authors have declared that they have no financial relationships at present or within the previous three years with any organizations that might have an interest in the submitted work. Other relationships: All authors have declared that there are no other relationships or activities that could appear to have influenced the submitted work.

\section{References}

1. Almas T, Ehtesham M, Khan AW, et al.: Safety and efficacy of low-dose corticosteroids in patients with nonsevere coronavirus disease 2019: a retrospective cohort study. Cureus. 2021, 13:e12544. 10.7759/cureus.12544

2. Hashim L, Khan HR, Ullah I, et al.: Physician preparedness in response to the coronavirus disease 2019 pandemic: a cross-sectional study from a developing country. Cureus. 2020, 12:e10383. 10.7759/cureus.10383

3. Nicholson AG, Osborn M, Devaraj A, Wells AU: COVID-19 related lung pathology: old patterns in new clothing?. Histopathology. 2020, 77:169-72. 10.1111/his.14162

4. Sun Z, Zhang N, Li Y, Xu X: A systematic review of chest imaging findings in COVID-19 . Quant Imaging Med Surg. 2020, 10:1058-79. 10.21037/qims-20-564

5. Khaliq M, Raja R, Khan N, Hanif H: An analysis of high-resolution computed tomography chest 
manifestations of COVID-19 patients in Pakistan. Cureus. 2020, 12:e9373. 10.7759/cureus.9373

6. Dai H, Zhang X, Xia J, et al.: High-resolution chest CT features and clinical characteristics of patients infected with COVID-19 in Jiangsu, China. Int J Infect Dis. 2020, 95:106-12. 10.1016/j.ijid.2020.04.003

7. Guan CS, Lv ZB, Yan S, et al.: Imaging features of coronavirus disease 2019 (COVID-19): evaluation on thinsection CT. Acad Radiol. 2020, 27:609-13. 10.1016/j.acra.2020.03.002

8. Francone M, Iafrate F, Masci GM, et al.: Chest CT score in COVID-19 patients: correlation with disease severity and short-term prognosis. Eur Radiol. 2020, 30:6808-17. 10.1007/s00330-020-07033-y

9. Grassi R, Fusco R, Belfiore MP, et al.: Coronavirus disease 2019 (COVID-19) in Italy: features on chest computed tomography using a structured report system. Sci Rep. 2020, 10:17236. 10.1038/s41598-02073788-5

10. Awulachew E, Diriba K, Anja A, Getu E, Belayneh F: Computed tomography (CT) imaging features of patients with COVID-19: systematic review and meta-analysis. Radiol Res Pract. 2020, 2020:1023506. $10.1155 / 2020 / 1023506$

11. Li K, Wu J, Wu F, Guo D, Chen L, Fang Z, Li C: The clinical and chest CT features associated with severe and critical COVID-19 pneumonia. Invest Radiol. 2020, 55:327-31. 10.1097/RLI.0000000000000672

12. Bao C, Liu X, Zhang H, Li Y, Liu J: Coronavirus disease 2019 (COVID-19) CT findings: a systematic review and meta-analysis. J Am Coll Radiol. 2020, 17:701-9. 10.1016/j.jacr.2020.03.006

13. Zayed NE, Bessar MA, Lutfy S: CO-RADS versus CT-SS scores in predicting severe COVID-19 patients: retrospective comparative study. Egypt J Bronchol. 2021, 15:13. 10.1186/s43168-021-00060-3

14. Zhou S, Wang Y, Zhu T, Xia L: CT features of coronavirus disease 2019 (COVID-19) pneumonia in 62 patients in Wuhan, China. AJR Am J Roentgenol. 2020, 214:1287-94. 10.2214/AJR.20.22975

15. Majidi H, Bani-Mostafavi ES, Mardanshahi Z, Godazandeh F, Ghasemian R, Heydari K, Alizadeh-Navaei R: High-resolution computed tomography finding in 552 patients with symptomatic COVID-19: first report from north of Iran. Emerg Radiol. 2020, 27:633-9. 10.1007/s10140-020-01819-9

16. Bernheim A, Mei X, Huang M, et al.: Chest CT findings in coronavirus disease-19 (COVID-19): relationship to duration of infection. Radiology. 2020, 295:200463. 10.1148/radiol.2020200463

17. Chen N, Zhou M, Dong X, et al.: Epidemiological and clinical characteristics of 99 cases of 2019 novel coronavirus pneumonia in Wuhan, China: a descriptive study. Lancet. 2020, 395:507-13. 10.1016/S01406736(20)30211-7

18. Korkmaz I, Dikmen N, Keleş FO, Bal T: Chest CT in COVID-19 pneumonia: correlations of imaging findings in clinically suspected but repeatedly RT-PCR test-negative patients. Egypt J Radiol Nucl Med. 2021, 52:96. 10.1186/s43055-021-00481-6

19. Yusuf S, Ahmad H, Zeb R, Zeb U, Zeb AA: High-resolution CT chest findings in suspected COVID-19 pneumonia patients with negative real-time polymerase chain reaction assay. Cureus. 2021, 13:e14023. 10.7759 /cureus. 14023 